\title{
Estado de salud e intención de volver a Estados Unidos en migrantes mexicanos: ¿evidencia de un sesgo de retorno selectivo?
}

\author{
Ietza Bojórquez Chapela* \\ Eduardo González Fagoaga** \\ Tonatiuh Guillén López***
}

El objetivo de este trabajo ha sido explorar la asociación entre la intención de permanecer en México (versus la intención de volver a Estados Unidos), con los indicadores del estado de salud de los migrantes que entraron a México por la frontera norte en 2010, para lo cual se utilizó información de la Encuesta de Migración en la Frontera Norte (Emif-N). Fue menor la probabilidad de que desearan volver a Estados Unidos quienes manifestaron peor estado de salud, en comparación quienes reportaron mejor estado de salud.

Palabras clave: migración internacional, migrantes, México, paradoja hispana.

Fecha de recepción: 7 de mayo de 2012.

Fecha de aceptación: 22 de marzo de 2013.

Health Status and Intention to Return to the United States in Mexican Immigrants: Evidence of a Selective Return Bias?

The aim of this study was to explore the association between the intention of remaining in Mexico (versus the intention of returning to the United States), with indicators on the health status of migrants who entered Mexico through the northern border in 2010, for which information from the Northern Border Migration Survey (Emif-N) was used. Those with a worse state of health were less likely to want to return to the United States compared with those in better state of health.

Key words: international migration, migrants, Mexico, Hispanic paradox.

* Departamento de Estudios de Población, El Colegio de la Frontera Norte, Tijuana. Correo electrónico: <ietzabch@colef.mx>. colef.mx>.

** El Colegio de la Frontera Norte, Tijuana. Correo electrónico: <eduardog@

*** Presidencia, El Colegio de la Frontera Norte, Tijuana. Correo electrónico: <presidencia@colef.mx>.

$\overline{\text { ESTUDIOS DEMOGRÁFICOS Y URBANOS, VOL. 29, NÚM. } 1 \text { (85), 2014, 165-182 }}$ 


\section{Introducción}

La "paradoja hispana", planteada por primera vez por Markides y Coreil en 1986 (Markides y Eschbach, 2005), consiste en la observación de que en Estados Unidos los grupos denominados "hispanos" presentan perfiles de salud iguales o incluso mejores que los de los blancos no hispanos, a pesar de sus peores condiciones económicas, de su menor acceso a los servicios de atención en salud, y de la mayor prevalencia entre ellos de afectaciones como diabetes y obesidad (Lerman Garber, Villa y Caballero, 2004; Markides y Eschbach, 2005; Turra y Goldman, 2007). Esta situación se ha observado mediante diversos indicadores de salud, como la mortalidad de niños y adultos, el peso al nacer, o la hipertensión arterial, entre otros (Palloni y Morenoff, 2001; Riosmena, Wong y Palloni, 2012). Sin embargo, la aparente ventaja de los hispanos en términos de salud no es general, sino específica de ciertos grupos y comparaciones. Así, la "paradoja” es más evidente al comparar las diferencias de la mortalidad de los mexicano-americanos y de los blancos no hispanos, sobre todo entre los hombres y en la población de mayor edad (Markides y Eschbach, 2005; Riosmena, Wong y Palloni, 2012). Se ha reportado también que la menor mortalidad de los hispanos es más notable entre personas con menor nivel educativo, mientras que entre aquellos con nivel educativo mayor hay más mortalidad en los hispanos que en los blancos no hispanos (Turra y Goldman, 2007). Asimismo, las diferencias en salud parecen ser más pronunciadas entre los hispanos nacidos fuera de Estados Unidos y los blancos no hispanos, mientras que los hispanos nacidos dentro del país muestran peor estado de salud que los blancos no hispanos (Palloni y Arias, 2004; Riosmena, Wong y Palloni, 2012).

La ventaja hispana en mortalidad ha recibido diferentes explicaciones. Primero, aunque una gran cantidad de estudios ha descrito este fenómeno, en algunos la diferencia desaparece una vez ajustando por otros factores que podrían estar asociados. Así, en un estudio de cohorte se encontró que la mortalidad por todas las causas fue mayor entre los hispanos que entre los blancos no hispanos, después de ajustar por diabetes, hipertensión y otras covariables (Hunt et al., 2003). En el mismo sentido, la prevalencia de factores de riesgo asociados a la mortalidad, como la presión arterial elevada o los componentes del síndrome metabólico, es similar o mayor en los hispanos que en los blancos no hispanos en Estados Unidos después de ajustar por el nivel socioeconómico, por lo que sería poco razonable esperar que el primer 
grupo presentara menor mortalidad (Crimmins, Kim, Alley, Karlamangla y Seeman, 2007).

Se ha sugerido también que la paradoja podría ser un artefacto metodológico ocasionado por errores diferenciales en el registro de la etnicidad entre los censos y los certificados de defunción, las dos principales fuentes utilizadas para estimar la mortalidad. En el caso de los censos, se registra la etnicidad por autorreporte, mientras que en el certificado de defunción el registro depende de quién lo elabora, y como resultado, en los certificados de defunción podría haber un subregistro de la etnicidad hispana. Otra posible fuente de sesgo de información sería la tendencia de los hispanos de edades avanzadas a reportarse en los censos como mayores de lo que son, lo cual incrementaría artificialmente la población en esos grupos de edad. Ambas situaciones resultarían en una subestimación de las tasas de mortalidad entre los hispanos. Adicionalmente, en las bases de datos con información individual es más frecuente que los hispanos se pierdan al seguimiento, en comparación con los blancos no hispanos. Sin embargo, los estudios que han buscado cuantificar la magnitud de estos problemas concluyen en general que el sesgo de información, si bien existe, no explica por completo la ventaja en mortalidad de los hispanos (Palloni y Arias, 2004; Patel, Eschbach, Ray y Markides, 2004; Waldstein, 2010).

Otros autores aseguran que la ventaja a favor de los hispanos es real, y se debe a aspectos protectores de la cultura hispana. Entre estos factores podrían estar las prácticas de autocuidado y la menor prevalencia de tabaquismo entre otros (Blue y Fenelon, 2011). El efecto protector de la cultura podría perderse con el tiempo transcurrido desde la inmigración, e incluso revertirse, lo que explicaría que la paradoja hispana aplique más a los inmigrantes de primera generación que a los hispanos nacidos en Estados Unidos (Turra y Goldman, 2007).

Por otra parte, la paradoja hispana podría ser resultado de las características de la migración a Estados Unidos, y del retorno a los lugares de origen. La hipótesis del migrante sano propone que quienes llegan a Estados Unidos tienen en general mejor salud que quienes permanecen en sus países de origen, por lo que la población de hispanos en ese país es de inicio una población más saludable (Crimmins et al., 2007; Riosmena, Wong y Palloni, 2012; Turra y Goldman, 2007). La segunda hipótesis corresponde a un sesgo de selección por el retorno diferencial a los lugares de origen (el llamado "sesgo del salmón”), en el que los migrantes enfermos regresan a su país en busca de atención médica o de condiciones de mayor apoyo social. 
Al salir de la población de Estados Unidos los migrantes enfermos, los registros en ese país tienden a incluir sólo a los más saludables (Palloni y Arias, 2004; Riosmena, Wong y Palloni, 2012; Turra y Goldman, 2007). La evidencia en este sentido no es aún concluyente. Por una parte, de manera consistente con el sesgo de retorno selectivo, se ha observado que la diferencia en mortalidad favorece más a los hispanos nacidos fuera de Estados Unidos, quienes en caso de enfermar tendrían más probabilidades de regresar a sus países de origen. Los hispanos nacidos en Estados Unidos, al carecer de redes de apoyo social en el país de origen, no irían allá al enfermar, por lo que no se esperaría observar en Estados Unidos una diferencia entre su salud y la de los blancos no hispanos (Palloni y Arias, 2004; Turra y Goldman, 2007). Sin embargo, algunos estudios sugieren que la magnitud del sesgo sería demasiado pequeña para explicar por completo la paradoja (Turra y Elo, 2008).

Con la intención de contribuir a la discusión del fenómeno de la paradoja hispana, este artículo tiene como objetivo explorar la asociación entre la condición de salud y la intención de volver posteriormente a Estados Unidos, versus permanecer en México. Para ello, se utilizó la información proporcionada por la Encuesta sobre Migración en la Frontera Norte de México (Emif-N), una fuente poco explorada en este sentido, y que tiene la ventaja de hacer preguntas acerca de las razones del retorno al país y la intención de volver posteriormente a Estados Unidos.

\section{Material y métodos}

\section{Muestra}

Se utilizó para el análisis la información de la Emif-N en el levantamiento de 2010. Ésta es una fuente de información representativa de los flujos migratorios en 12 ciudades de la frontera México-Estados Unidos, y en cuatro ciudades más que son sitios de tránsito para los migrantes entre estos países. La metodología detallada de la Emif ha sido descrita previamente (El Colegio de la Frontera Norte, 2011). En síntesis, se trata de una encuesta basada en técnicas de muestreo probabilístico para poblaciones móviles, que selecciona aleatoriamente puntos de espacio y tiempo en los que se recoge información, entre otros, de los migrantes mexicanos que regresan de Estados Unidos. 
Los puntos de levantamiento corresponden a sitios de cruce fronterizo, terminales de autobuses y aeropuertos en 12 ciudades fronterizas, y aeropuertos en cuatro ciudades más del interior del país, elegidas por su elevado flujo de migrantes. Se aplica la encuesta únicamente a personas de 15 años o más que no hayan nacido en Estados Unidos. Los levantamientos se realizan de manera continua durante todo el año. Su propósito principal es recabar datos estadísticos con el fin de proporcionar información para la toma de decisiones en políticas públicas, y no recoge información que permita la identificación personal.

En la edición 2010 de la Emif-N, a los encuestados que regresaban a México procedentes de Estados Unidos se les aplicó un cuestionario con preguntas acerca de su estado de salud. Para el presente trabajo se utilizaron los datos del primer trimestre, una muestra de 5850 personas que, una vez aplicados los factores de ponderación, representan a 440972 individuos. Considerando que la evidencia a favor o en contra del sesgo de retorno selectivo requería que la persona hubiera permanecido en Estados Unidos el tiempo suficiente para ser contada como parte de su población, se excluyó a quienes habían estado en ese país por menos de tres meses. Se excluyó también a quienes habían sido devueltos a México por la patrulla fronteriza de Estados Unidos, ya que se consideró que en este caso el regreso no era voluntario y no estaría influenciado por el estado de salud. La muestra para el análisis quedó compuesta por 3663 personas $(72.5 \%$ de la muestra, una vez aplicado el factor de ponderación) (esquema 1).

\section{Definición de variables}

La variable dependiente en este análisis fue la intención de volver a Estados Unidos versus la intención de permanecer en México. Se construyó esta variable de dos maneras diferentes y los análisis se llevaron a cabo con ambas formas. En la primera, a partir de la pregunta "Principalmente, ¿por qué razón regresa usted a México?”, se consideró que el encuestado pensaba volver a Estados Unidos si respondía que su principal razón para estar en México era visitar familiares o amigos, o participar en alguna festividad, lo que daría un carácter temporal a la visita. Se consideró que el encuestado tenía intención de permanecer definitivamente en México si reportaba como su principal razón la falta de trabajo en Estados Unidos, o ingresos insuficientes, o mala 


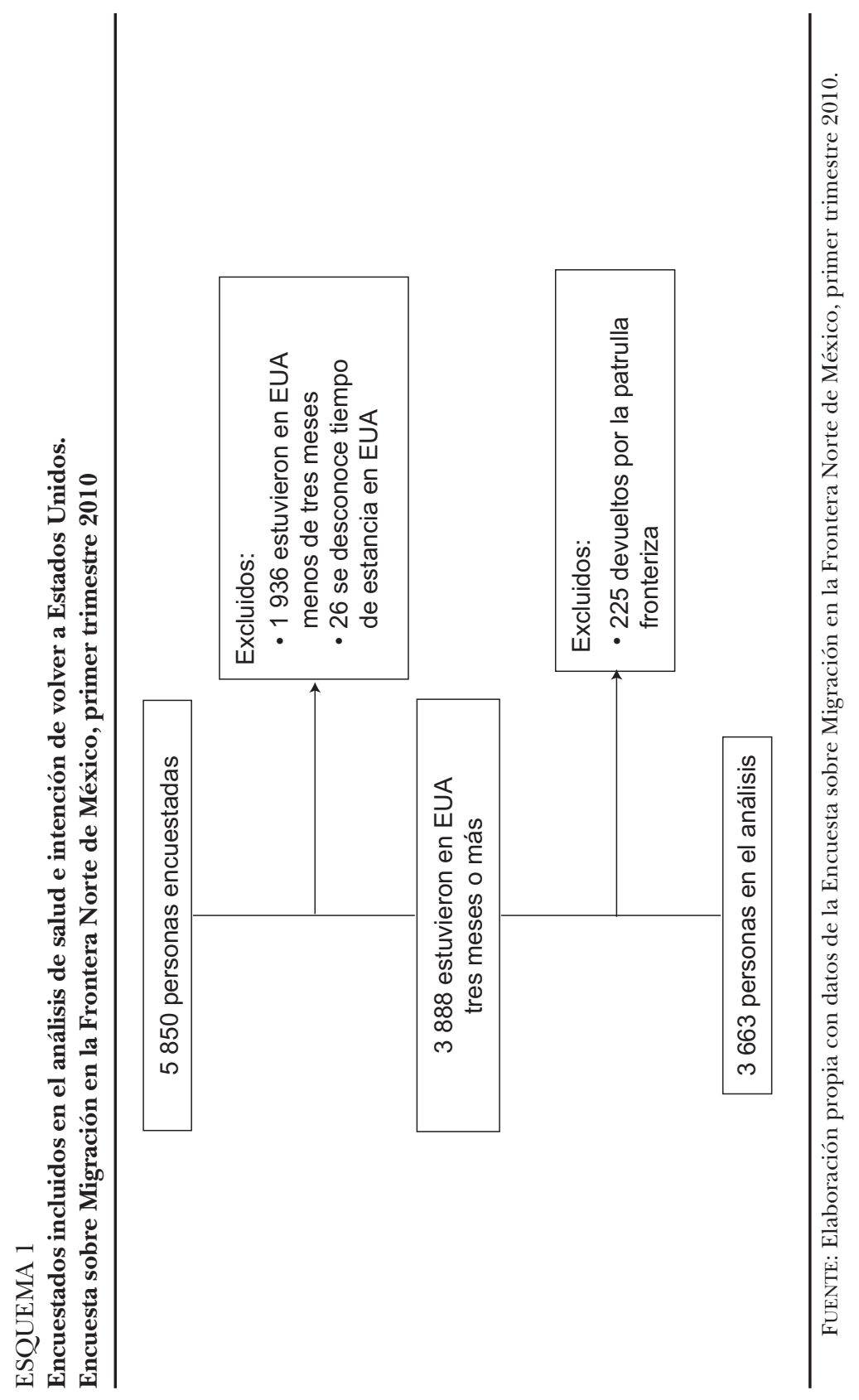


adaptación en ese país, o la intención de vivir o trabajar en México, o la de retirarse o jubilarse.

En la segunda forma de la variable se clasificó a los encuestados de acuerdo con la pregunta "¿Piensa volver algún día a Estados Unidos a trabajar o a buscar trabajo?”, con opciones de respuesta sí/no.

La principal variable independiente considerada fue el estado de salud, según lo percibía el encuestado, clasificado en dos categorías: muy buena/buena (codificada como " 0 ") versus regular/mala/muy mala (codificada como "1"). La autopercepción de salud es una medida ampliamente utilizada en estudios de salud pública que ha demostrado ser un buen predictor de la mortalidad (Quesnel Vallee, 2007; Yu et al., 1998). Se utilizaron también el autorreporte de diagnóstico de diabetes e hipertensión, y la presencia de síntomas de malestar emocional. Estos últimos se evaluaron por la respuesta (dicotómica) a las preguntas: "Durante su estancia en Estados Unidos, ¿frecuentemente se sintió deprimido/triste?", "Durante su estancia en Estados Unidos, ¿frecuentemente se sintió nervioso/intranquilo/desesperado?" y "Durante su estancia en Estados Unidos, ¿frecuentemente se sintió cansado/agotado?". Se consideró que el encuestado presentaba malestar emocional si respondía de manera afirmativa a dos o más de estas preguntas.

Finalmente, como potenciales confusores de la asociación entre el estado de salud y la intención de retorno se consideraron la edad, el sexo, el nivel educativo, el estado conyugal, y el hablar una lengua indígena como indicador de pertenencia a un grupo indígena. Como covariables de interés se incluyeron en el análisis el tiempo en Estados Unidos, el ser ciudadano estadounidense, y el contar o no con trabajo o seguro médico en ese país.

\section{Análisis estadístico}

Se utilizó el modelo de regresión logística para evaluar las asociaciones entre la variable de respuesta (intención de volver a Estados Unidos), los indicadores de salud y las covariables de interés. Se ajustaron modelos simples para cada asociación, y modelos multivariados. Para la elaboración de estos últimos se analizaron los resultados de los modelos simples, y su modificación al incluir covariables y posibles confusores. Se dejaron fuera del modelo final aquellas variables que, o bien no mostraron asociación estadísticamente significativa con la 
variable dependiente en los modelos multivariados, o bien disminuían el ajuste del modelo. Considerando que las condiciones de salud y otras covariables podrían tener un efecto diferente sobre los hombres que sobre las mujeres, los modelos se estratificaron por sexo. Al no observarse diferencias importantes, se decidió presentar los resultados de manera conjunta para toda la muestra. En todos los análisis se tomaron en cuenta los ponderadores correspondientes al diseño de muestra, y los errores estándar se ajustaron mediante estimación de la varianza linearizada de Taylor. Todos los análisis se llevaron a cabo con el módulo Svy del programa Stata/SE versión 11.2 (Stata Corp., College Station, Tx., Estados Unidos). Los resultados se presentan con las cifras considerando los ponderadores, a menos que se indique lo contrario.

\section{Resultados}

De los encuestados incluidos en el análisis, 81.7\% (IC95\% 79.6, 83.9) tenía intención de volver a Estados Unidos, y 84.3\% (IC95\% 82.4, 86.3) tenía intención de volver a trabajar en ese país. En el cuadro 1 se muestran las características de los encuestados, de acuerdo con su intención de volver a Estados Unidos. Quienes tenían intención de permanecer en México eran en general más jóvenes, un mayor porcentaje hablaba lengua indígena, y un menor porcentaje estaba casado o en unión. Este grupo tuvo también la peor salud percibida y mayor frecuencia de síntomas de malestar emocional. Por otra parte, el porcentaje de personas con ciudadanía estadounidense fue mucho menor $(6.0 \%)$ en comparación con quienes tenían la intención de volver a ese país $(38.8 \%)$, lo mismo que el porcentaje de personas con empleo (18.9 versus $71.4 \%$ ) y seguro médico en Estados Unidos (16.7 versus $45.5 \%)$. De acuerdo con esta información, y de manera congruente con la hipótesis del retorno selectivo, quienes salen de Estados Unidos para permanecer en México muestran peor estado de salud, en comparación con los que piensan volver a Estados Unidos.

Para evaluar la asociación entre la salud y la intención de volver a Estados Unidos, se estimaron las razones de momios (RM) de intención de volver a ese país, comparando a los grupos de acuerdo con los indicadores del estado de salud, después de ajustar por otras variables que podrían confundir la asociación (cuadros 2 y 3). En los modelos finales se observó una menor probabilidad de querer volver a Estados 
Unidos entre quienes percibían su salud como regular, mala o muy mala, en comparación con quienes la percibían como buena o muy buena, y quienes tenían síntomas de malestar emocional también tuvieron menor probabilidad de querer volver a Estados Unidos. Las personas de mayor edad, las que estaban casadas o tenían una pareja estable, los ciudadanos estadounidenses, los individuos con empleo y seguro médico en Estados Unidos, fueron quienes tuvieron mayor probabilidad de haber entrado a México sólo temporalmente. En los modelos estratificados por sexo las direcciones de las asociaciones fueron las mismas, aunque entre los hombres el estado conyugal, la salud percibida y el malestar emocional no se asociaron de manera significativa con la intención de volver a Estados Unidos (no se muestran en los cuadros).

\section{Discusión}

El objetivo central de este estudio fue evaluar la asociación entre el estado de salud y la intención de volver a Estados Unidos, en una muestra de personas que ingresaron a México después de haber permanecido en Estados Unidos durante tres meses o más. La dirección de las asociaciones mostró de manera consistente que una mejor salud percibida y la ausencia de síntomas de malestar emocional aumentan la probabilidad de querer volver a Estados Unidos. Si bien la intención de volver no asegura que en efecto la persona regresará a Estados Unidos en un futuro cercano, los resultados de este análisis apuntan a la existencia de un fenómeno de retorno selectivo, donde quienes salen de Estados Unidos de manera permanente se encuentran en peores condiciones de salud, en comparación con quienes lo hacen de manera temporal.

Aunque la muestra analizada no permite llegar a conclusiones acerca de la mortalidad, y se trata de un grupo relativamente joven (promedio de edad de 42 años), quienes respondieron en la encuesta que su salud era mala o muy mala podrían presentar en el futuro problemas de salud. Si este grupo efectivamente permanece en México, en lugar de volver a Estados Unidos, la población de ese país estaría perdiendo selectivamente a un grupo de hispanos con peor salud, como sugiere la hipótesis del "sesgo del salmón". Por su parte, el malestar emocional también se liga a la salud física, así como a las conductas de prevención de enfermedades (World Health Organization, 


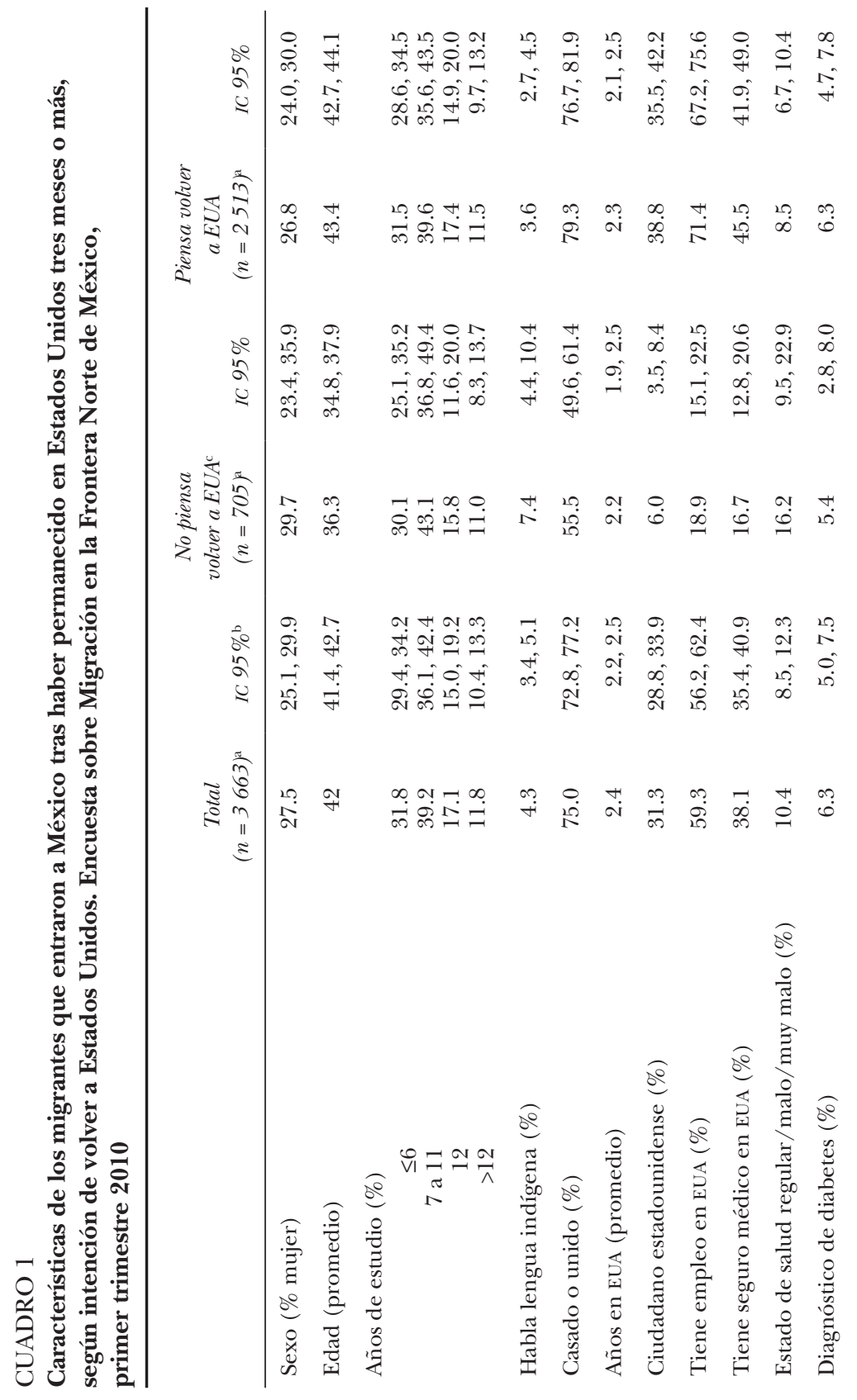




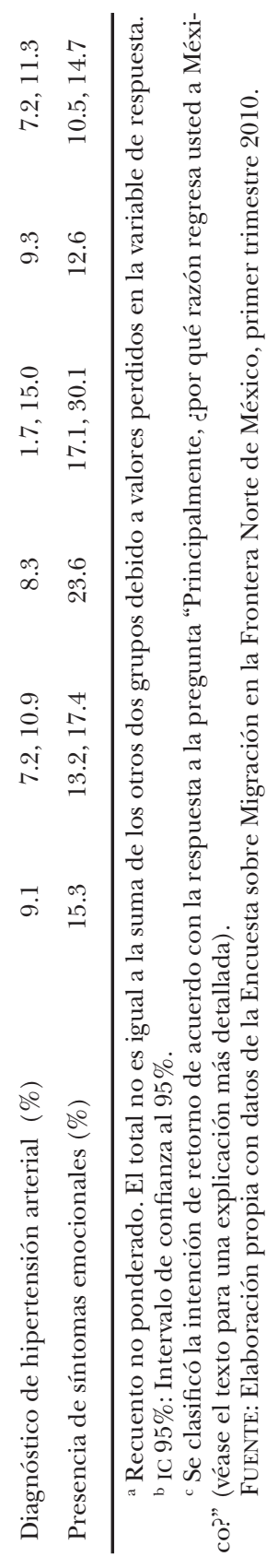


CUADRO 2

Características asociadas a la intención de volver a Estados Unidos entre los migrantes que entraron a México tras haber permanecido en Estados Unidos tres meses o más. ${ }^{a}$ Encuesta sobre Migración en la Frontera Norte de México, primer trimestre $2010(n=3$ 017)

\begin{tabular}{lcr}
\hline & $R M^{\mathrm{b}}$ & $I C 95 \%^{\mathrm{c}}$ \\
\hline Edad (años) & 1.03 & $1.02,1.05$ \\
Años en EUA & 1.04 & $1.0,1.09$ \\
Estar casado o unido & 1.93 & $1.38,2.70$ \\
Ser ciudadano estadounidense & 7.75 & $4.53,13.23$ \\
Tener empleo en Estados Unidos & 8.97 & $6.61,12.18$ \\
Tener seguro médico en Estados Unidos & 1.70 & $1.21,2.39$ \\
Estado de salud regular / malo / muy malo & 0.56 & $0.35,0.90$ \\
Presencia de síntomas emocionales & 0.68 & $0.48,0.97$ \\
\hline
\end{tabular}

${ }^{a}$ Resultados de regresión logística ajustada por las demás variables en el modelo. Se clasificó la intención de retorno de acuerdo con la respuesta a la pregunta "Principalmente, ¿por qué razón regresa usted a México?" (véase el texto para una explicación más detallada).

b RM: Razón de momios.

${ }^{\mathrm{c}}$ IC95\%: Intervalo de confianza al 95\%.

FUENTE: Elaboración propia con datos de la Encuesta sobre Migración en la Frontera Norte de México, primer trimestre 2010.

WHO, 2001), por lo que la observación de que quienes tenían mayor malestar emocional eran los más inclinados a quedarse en México también apoya la hipótesis del retorno selectivo.

En cambio, el porcentaje de quienes reportaron haber recibido un diagnóstico de diabetes o hipertensión fue muy similar entre los grupos de acuerdo con la intención de volver a Estados Unidos. Esta ausencia de asociación podría deberse en parte a que se analizó información de personas que en su mayoría se encontraban en edades previas a aquellas en que suelen desarrollarse dichos padecimientos. Es posible también que la asociación estuviera confundida por el efecto del acceso a servicios médicos. En la población mexicana (M.E. Garza Elizondo et al., 2008; M.F. Garza Elizondo, Villarreal Ríos, Salinas Martinez y Nuñez Rocha, 2004; Pagan, Puig y Soldo, 2007) e hispana en Estados Unidos (Liao et al., 2011; Salgado de Snyder, González, Bojórquez e Infante, 2007) las enfermedades crónicas pueden transcurrir sin diagnóstico por periodos prolongados debido principalmen- 


\section{CUADRO 3}

Características asociadas a la intención de volver a trabajar a Estados Unidos entre los migrantes que entraron a México tras haber permanecido en Estados Unidos tres meses o más. ${ }^{a}$ Encuesta sobre Migración en la Frontera Norte de México, primer trimestre 2010 ( $n=3$ 332)

\begin{tabular}{lcr}
\hline & $R M^{b}$ & $I C 95 \%^{c}$ \\
\hline Edad (años) & 1.00 & $0.99,1.02$ \\
Años en EUA & 1.03 & $0.99,1.07$ \\
Estar casado o unido & 1.63 & $1.15,2.30$ \\
Ser ciudadano estadounidense & 1.80 & $1.21,2.68$ \\
Tener empleo en Estados Unidos & 21.7 & $14.13,33.44$ \\
Tener seguro médico en Estados Unidos & 1.52 & $1.04,2.22$ \\
Estado de salud regular / malo / muy malo & 0.56 & $0.33,0.94$ \\
Presencia de síntomas emocionales & 0.51 & $0.34,0.75$ \\
\hline
\end{tabular}

${ }^{a}$ Resultados de regresión logística ajustada por las demás variables en el modelo. Se definió la intención de volver a EUA de acuerdo con la respuesta a la pregunta “¿Piensa volver algún día a Estados Unidos a trabajar o buscar trabajo?” (véase el texto para una explicación más detallada).

${ }^{\mathrm{b}} \mathrm{RM}$ : Razón de momios.

${ }^{c}$ IC95\%: Intervalo de confianza al 95\%.

FUENTE: Elaboración propia con datos de la Encuesta sobre Migración en la Frontera Norte de México, primer trimestre 2010.

te a la dificultad de acceso a esos servicios. Quienes no reportaron un diagnóstico de enfermedad crónica podrían ser los que carecían de acceso, no aquellos con mejor salud. Dado que éstos probablemente pertenecieran al grupo con menos recursos para permanecer en Estados Unidos, el resultado sería que personas aparentemente sanas tendrían menos intención de volver a ese país, sesgando la asociación hacia el valor nulo.

Por otro lado, de entre las variables consideradas las que mostraron una relación más consistente con la intención de retorno fueron las que pueden entenderse como indicadoras de las condiciones de vida, las oportunidades y el acceso a servicios en Estados Unidos: el ser ciudadano estadounidense y el tener allí empleo y seguro médico. Una vez ajustando por estas variables, el efecto del estado de salud percibida sobre la intención de volver disminuyó. El grado de estabilidad de la vida en Estados Unidos podría también modificar la relación entre el estado de salud y la intención de volver: algunos estudios han en- 
contrado que la paradoja hispana se aplica más a los nacidos fuera de Estados Unidos que a los hispanos nacidos en ese país (Palloni y Arias, 2004), lo que se explica si los segundos, al tener mejor acceso a servicios y en general a condiciones más estables, no requieren regresar a sus lugares de origen al enfermar.

La principal limitación de este estudio fue el uso de variables que son sólo indicadores aproximados, tanto de la probabilidad de salir de la población de Estados Unidos, como de la condición de salud. La variable dependiente considera únicamente la intención, que podría o no traducirse en el hecho de retornar a ese país. En la segunda forma de la variable, además, se presenta el problema de que se excluye a aquellos que piensan volver a Estados Unidos por razones diferentes de la búsqueda de empleo. Si bien el estado de salud autorreportado es un indicador imperfecto, ofrece una buena aproximación al estado físico (Quesnel Vallee, 2007; Yu et al., 1998). Sin embargo, la consistencia de las asociaciones entre los diversos análisis puede tomarse como apoyo a la existencia de una relación entre la salud y la intención de retorno.

Con el proceso de desaceleración económica que se ha registrado en Estados Unidos a partir de 2006, la migración de mexicanos hacia ese país ha comenzado a descender en forma continua hasta alcanzar en la actualidad niveles cercanos a la mitad de lo que se observó en 2007. Junto con este descenso y asociado a la profundización de la crisis se especuló respecto a la posibilidad de un retorno masivo de mexicanos desde Estados Unidos debido a los problemas de desaparición de empleos en ese país, así como a la crisis en el sector inmobiliario y en la industria de la construcción, entre otros sectores de la economía estadounidense en los que tradicionalmente se incorporan los migrantes mexicanos. No obstante lo anterior, los datos de la encuesta no muestran evidencias de retorno masivo, más allá del regreso que se ve incrementado de manera estacional durante los periodos de verano y de fiestas decembrinas (Alarcón et al., 2009). En trabajos futuros sería interesante explorar si las asociaciones observadas en este análisis se modifican a lo largo de los años y guardan relación con los cambios económicos.

El fenómeno de retorno selectivo en la población hispana es un tema cuyas características y motivos se encuentran aún en discusión (Riosmena, Wong y Palloni, 2012). Por otro lado, como han señalado otros autores (Wong y González González, 2010), desde el punto de vista del país al que retornan los migrantes, este fenómeno puede 
constituir un problema importante de salud pública, pues ingresan a éste personas que vuelven en condiciones de salud deterioradas después de haber cumplido su vida laboral útil en el extranjero. Desde ambos puntos de vista, el tema de la paradoja hispana merece una exploración continua.

\section{Bibliografía}

Alarcón, R., R. Cruz, A. Díaz Bautista, G. González König, A. Izquierdo, G. Irizar y R. Zenteno (2009), "La crisis financiera en Estados Unidos y su impacto en la migración mexicana”, Migraciones Internacionales, vol. 5, núm. 1, pp. 193-210.

Blue, L. y A. Fenelon (2011), "Explaining Low Mortality among US Immigrants Relative to Native-Born Americans: The Role of Smoking", International Journal of Epidemiolgy, vol. 40, núm. 3, pp. 786-793.

Crimmins, E.M., J.K. Kim, D.E. Alley, A. Karlamangla y T. Seeman (2007), "Hispanic Paradox in Biological Risk Rrofiles", American Journal of Public Health, vol. 97, núm. 7, pp. 1305-1310.

El Colegio de la Frontera Norte (2011), Encuesta sobre Migración en la Frontera Norte de México, 2009, México, Segob / SRE / STPS / El Colegio de la Frontera Norte.

Garza Elizondo, M.E., A.M. Salinas Martínez, G.M. Núñez Rocha, E. Villarreal Ríos, M.G. Vásquez Treviño y M.G. Vásquez Salazar (2008), “Accesibilidad geográfica para detección temprana de enfermedades crónico-degenerativas”, Revista Médica de Chile, vol. 136, núm. 12, pp. 1574-1581.

Garza Elizondo, M.F., E. Villarreal Ríos, A.M. Salinas Martínez y G.M. Núñez Rocha (2004), "Prácticas preventivas de los habitantes mayores de 25 años en Monterrey y su zona metropolitana (México)”, Revista Española de Salud Pública, vol. 78, núm. 1, pp. 95-105.

Hunt, K.J., R.G. Reséndez, K. Williams, S.M. Haffner, M.P. Stern y H.P. Hazuda (2003), "All-Cause and Cardiovascular Mortality among Mexican-American and Non-Hispanic White Older Participants in the San Antonio Heart Study. Evidence against the 'Hispanic Paradox'”, American Journal of Epidemiology, vol. 158, núm. 11, pp. 1048-1057.

Lerman Garber, I., A.R. Villa y E. Caballero (2004), "Diabetes and Cardiovascular Disease. Is There a True Hispanic Paradox?”, Revista de Investigación Clínica, vol. 56, núm. 3, pp. 282-296.

Liao, Y., D. Bang, S. Cosgrove, R. Dulin, Z. Harris, A. Taylor y W. Giles (2011), "Surveillance of Health Status in Minority Communities. Racial and Ethnic Approaches to Community Health across the U.S. (REACH U.S.) Risk Factor Survey, United States, 2009”, Morbidity and Mortality Weekly Report. Surveillance Summaries, vol. 60, no. 6, pp. 1-44. 
Markides, K.S. y K. Eschbach (2005), “Aging, Migration, and Mortality: Current Status of Research on the Hispanic Paradox", The Journal of Gerontology, Series B, Psychological Sciences and Social Sciences, vol. 60, núm. 2, pp. 68-75. Pagan, J.A., A. Puig y B.J. Soldo (2007), "Health Insurance Coverage and the Use of Preventive Services by Mexican Adults", Health Economics, vol. 16, núm. 12, pp. 1359-1369.

Palloni, A. y E. Arias (2004), "Paradox Lost: Explaining the Hispanic Adult Mortality Advantage”, Demography, vol. 41, núm. 3, pp. 385-415.

Palloni, A. y J.D. Morenoff (2001), "Interpreting the Paradoxical in the Hispanic Paradox: Demographic and Epidemiologic Approaches", Annals of the New York Academy of Sciences, vol. 954, núm. pp. 140-174.

Patel, K.V., K. Eschbach, L.A. Ray y K.S. Markides (2004), "Evaluation of Mortality Data for Older Mexican Americans: Implications for the Hispanic Paradox", American Journal of Epidemiology, vol. 159, núm. 7, pp. 707-715.

Quesnel Vallee, A. (2007), "Self-Rated Health: Caught in the Crossfire of the Quest for 'True' Health?”, International Journal of Epidemiology, vol. 36, núm. 6, pp. 1161-1164.

Riosmena, F., R. Wong y A. Palloni (2012), "Migration, Selection, Protection, and Acculturation in Health: A Binational Perspective on Older Adults", Demography, vol. 50, núm. 3, pp. 1039-1064.

Salgado de Snyder, N., T. González, I. Bojórquez y C. Infante (2007), Migración México-Estados Unidos: consecuencias para la salud, Cuernavaca, Instituto Nacional de Salud Pública.

Turra, C.M. e I.T. Elo (2008), "The Impact of Salmon Bias on the Hispanic Mortality Advantage: New Evidence from Social Security Data”, Population Research and Policy Review, vol. 27, núm. 5, pp. 515-530.

Turra, C.M. y N. Goldman (2007), "Socioeconomic Differences in Mortality among U.S. Adults: Insights into the Hispanic Paradox", The Journals of Gerontology. Series B, Psychological Sciences and Social Sciences, vol. 62, núm. 3, pp. S184-192.

Waldstein, A. (2010), "Popular Medicine and Self-Care in a Mexican Migrant Community: Toward an Explanation of an Epidemiological Paradox", Medical Anthropology, vol. 29, núm. 1, pp. 71-107.

Wong, R. y C. González González (2010), “Old-Age Disability and Wealth among Return Mexican Migrants from the United States", Journal of Aging and Health, vol. 22, núm. 7, pp. 932-954.

World Health Organization (WHO) (2001), The World Health Report 2001: Mental Health. New Understanding, New Hope, Ginebra, wHO.

Yu, E.S., Y.M. Kean, D.J. Slymen, W.T. Liu, M. Zhang y R. Katzman (1998), "Self-Perceived Health and 5-Year Mortality Risks among the Elderly in Shanghai, China”, American Journal of Epidemiology, vol. 147, núm. 9, pp. 880-890. 
Bojórquez, González y Guillén, ESTADO DE SALUD

\section{Acerca de los autores}

Ietza Bojórquez es doctora en Epidemiología por la Escuela de Salud Pública de México del Instituto Nacional de Salud Pública. Ha desarrollado diversos proyectos de investigación en los campos de la salud mental, las prácticas asociadas a la salud, y la relación entre las condiciones sociales y culturales y la salud. Es investigadora en el Departamento de Estudios de Población de El Colegio de la Frontera Norte, donde dirige los proyectos "Prácticas alimentarias, conductas alimentarias de riesgo y obesidad en un contexto de migración" y "Salud mental y factores asociados en personas deportadas a través de la frontera internacional en Baja California”.

Jesús Eduardo González Fagoaga es doctor en Ciencias Sociales, demógrafo y economista. Sus principales líneas de estudio son migración y salud, análisis demográfico, mortalidad y morbilidad relacionadas al $\mathrm{VIH} /$ sida, temas sobre los que ha publicado artículos en revistas nacionales y extranjeras. Ha colaborado en diversos proyectos relacionados con la migración internacional e interna, y el acceso a servicios de salud, entre otros. Imparte cursos a estudiantes de licenciatura, maestría y doctorado en instituciones públicas y privadas, tanto en México como en Guatemala.

Actualmente coordina el área de Estadística e Investigación de las Encuestas sobre Migración en las Fronteras Norte y Sur de México en El Colegio de la Frontera Norte.

Tonatiuh Guillén López es doctor en Ciencias Sociales con especialidad en Sociología por El Colegio de México. Actualmente es Presidente de El Colegio de la Frontera Norte (2012-2017), en donde ha sido director del Departamento de Estudios de Administración Pública y director de la revista académica Frontera Norte. Ha sido profesor en la Universidad Nacional Autónoma de México, la Universidad Autónoma de Coahuila y la Universidad Autónoma de Chiapas, además de profesor visitante en otras instituciones internacionales. Sus temas de investigación se centran en los estudios de política regional, modernización de los gobiernos locales, federalismo y descentralización. Es autor y coordinador de 15 libros y más de 60 capítulos de libros y artículos en revistas académicas. Es miembro del Sistema Nacional de Investigadores. Ha sido miembro del Foro Consultivo Científico y Tecnológico; presidente del Consejo Consultivo de Centros Públicos de Investigación 
ESTUDIOS DEMOGRÁFICOS Y URBANOS, VOL. 29, NÚM. 1 (85), 2014, 165-182

del Consejo Nacional de Ciencia y Tecnología (Conacyt), en donde además ha coordinado el Comité de Ciencias Sociales y Economía; fue presidente de la Red de Investigadores en Gobiernos Locales Mexicanos (Iglom, 2004-2007); y coordinador ejecutivo del Premio Gobierno y Gestión Local (2005 y 2006). 